\title{
Répression et activation des rétrovirus murins dans les cellules totipotentes
}

Les virus de leucémie murine sont transcriptionnellement inactifs dans les cellules embryonnaires où ils est soumis à plusieurs mécanismes de contrôle négatif. Cependant, dans de très rares cas, ces virus peuvent être intégrés à une unité de transcription cellulaire active et exprimés dans les cellules indifférenciées. Un transcrit hybride commençant au début d'un gène cellulaire se poursuit dans l'ADN proviral et est épissé en utilisant un site accepteur cryptique du virus. Un tel mécanisme pourrait aboutir à l'annexion par un rétrovirus de séquences cellulaires en aval desquelles il est intégré. De plus, la transcription de tout ou partie d'un génome viral, par lui-même inactif, est aussi probablement en cause dans le mécanisme de plusieurs affections virales.

Jean-François Nicolas Claire Bonnerot

ADRESSE

J.-F Nicolas : directeur de recherche à l'Inserm, chef d'unité à l'Institut Pasteur, codirecteur de l'URA 1148 du Cnrs. C. Bonnerot : chargée de recherche à l'Inserm. Unitć de biologie molćculaire du développement, Institut Pasteur, URA Cnrs 1148, 25, rue du Docteur-Roux, 75724 Paris Cedex 15, France.

$\mathrm{m} / \mathrm{s} n^{\circ} 2$ vol. 9 , férier 93 es rétrovirus murins peuvent infecter la plupart des types cellulaires. Cependant, ils ne se propagent que dans les cellules différenciées. Le blocage du cycle rétroviral dans les cellules indifférenciées (multipotentielles) est analysée à l'aide des cellules souches de carcinome embryonnaire (EC), cellules qui ont gardé beaucoup de propriétés biologiques des cellules multipotentielles de l'embryon dont elles dérivent [1]. Cettc analyse donne des informations non seulement sur les propriétés des rétrovirus, mais aussi sur les mécanismes de la différenciation cellulaire. Elle permet aussi dc décrire les stratégies utilisées par les rétrovirus murins pour passer inaperçus et pour détourner à leur profit des éléments du génome de la ccllulc infectée.

\section{Niveaux de blocage des rétrovirus dans les cellules EC}

Le blocage du cycle rétroviral dans les cellules multipotentielles est transcriptionnel. Le virus pénètrc dans les cellules et s'incorpore à leur génomc, 


\section{RÉFÉRENCES}

1. Robertson EJ. Teratocarcinomas and Embryonic Stem Cells. A Pratical Approach. Oxford : IRL Press, 1987.

2. Linney E, Davis B, Overhauser J, Chao E, Fan H. Non-function of a Moloney murine leukaemia virus regulatory sequence in F9 embryonal carcinoma cells. Nature $1984 ; 308: 470-2$

3. Speck NA, Baltimore D. Six distinct nuclear factors interact with the 75-base-pair repeat of the Moloney murine leukemia virus enhancer. Mol Cell Biol 1987; 7 : 1101-10.

4. Tsukiyama $\mathrm{T}$, Niwa $\mathrm{O}$, Yokoro $\mathrm{K}$. Analysis of the binding proteins and activity of the long terminal repeat of Moloney murine leukemia virus during differentiation of mouse embryonal carcinoma cells. J Virol $1991 ; 65$ : 2979-86.

5. Akgün E, Ziegler M, Grez M. Determinants of retrovirus gene expression in embryonal carcinoma cells. J Virol 1991; $65: 382-8$.

6. Grez M M Z, Nowock J, Ziegler M. A single point mutation activates the Moloney murine leukemia virus long terminal repeat in embryonal stem cells. J Virol $1991 ; 65$ : 4691-8.

7. Prince VE, Rigby PWJ. Derivatives of Moloney murine sarcoma virus capable of being transcribed in embryonal carcinoma stem cells have gained a functional Sp1 binding site. J Virol 1991; 65 : 1803-11.

8. Petersen R, Kempler G, Barklis E. A stem cell-specific silencer in the primerbinding site of a retrovirus. Mol Cell Biol 1991 ; $11: 1214-21$

9. Loh TP, Sievert LL, Scott RW. Evidence for a stem cell-specific repressor of Moloney murine leukemia virus expression in embryonal carcinoma cells. Mol Cell Biol $1990 ; 10$ : 4045-57.

10. Grez E, Akgün E, Hilbert F, Ostertag W. Embryonic stem cell virus, a recombinant murine retrovirus with expression in embryonic stem cells. Proc Natl Acad Sci USA $1990 ; 87$ : 9202-6. sous la forme d'un provirus, comme il le fait dans les autres types cellulaires. Mais il n'y est ensuite jamais transcrit [2]. La propagation de ce virus à ARN est donc arrêtée. Des expériences de transfection dans des cellules en culture où l'activité transcriptionnelle conférée par des éléments du virus à un gène rapporteur est mesurée, ont montré que trois éléments assurent le verrouillage du virus (figure 1).

1. Tout d'abord, la région activatrice du promoteur viral contenue dans la longue répétition terminale (LTR) 5' n'est pas fonctionnelle. Ce caractère non fonctionnel est dû à la fois à l'absence de facteurs transcriptionnels activateurs et à la présence de répresseurs spécifiques. Ainsi, parmi les facteurs qui se fixent normalement sur la région activatrice du MoMLV (Moloney murine leukemia virus), deux des principaux, NF-1 et le facteur nucléaire reconnaissant le motif central de SV40 (TGTGGTAT), sont absents des cellules EC [3]. De plus, un facteur répresseur se fixe sur la région - 354 à 306 du LTR du virus sauvage [4,5]. (Dans la numérotation utilisée ici, +1 correspond au premier nucléotide transcrit, voir légende de la figure 1). Les virus mutés dans cette région qui ont une activité transcriptionnelle dans les cellules multipotentielles ne fixent plus ce répresseur. L'ADNc du répresseur, appelé ELP [4] ou ECF-1 [5], a été cloné. Son expression n'est détectée que dans les cellules multipotentielles. Enfin, une mutation ponctuelle à la position - 166 du LTR est suffisante, en l'absence d'un site répresseur dénommé RBS (voir ci-après) pour activer le LTR dans les cellules multipotentielles [6, 7]. Cette mutation crée une séquence consensus pour le facteur de transcription Sp1, ce qui compense l'absence, notée plus haut, d'activateur transcriptionnel pour la région activatrice du LTR.

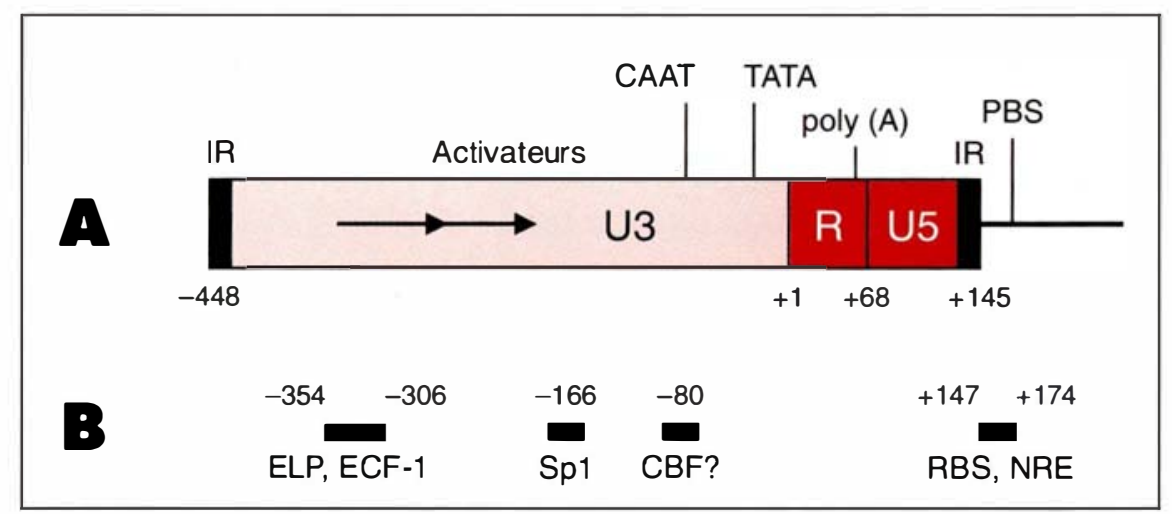

Figure 1. Éléments impliqués dans la répression des provirus dans les cellules multipotentielles. A. Le LTR du virus MoMLV est composé de trois régions : U3, $R$ et $U 5$. La région $R$ est répétée aux deux extrémités de l'ARN génomique. Le promoteur (boîtes TATA et CAAT) et la région activatrice sont dans la région U3. L'extrémité $5^{\prime}$ de la région $R$ située à 20 paires de base de la boîte TATA correspond au début de la transcription. Le premier nucléotide de l'ARN viral est numéroté + 1 . II correspond à la première paire de bases de $R$. La région PBS (pour primer binding site) correspond à une région où, sur l'ARN génomique, se trouve apparié un ARNt proline qui est utilisé comme amorce par la transcriptase inverse pour synthétiser le brin ADN + . B. Les régions impliquées dans la contrôle transcriptionnel négatif dans les cellules multipotentielles. Une région en amont de la région activatrice 1- 354 - 306), dans la région U3, fixe un facteur répresseur (ELP, ECF-1). La région - 166 correspond à des séquences qui, une fois mutées en un site $S p 1$, peuvent activer le LTR. La boîte CAAT pourrait fixer des facteurs inhibiteurs de la transcription (CBF). La région PBS fixe un silencer transcriptionnel (RBS, NRE) ainsi que $d^{\prime}$ autres facteurs (moins bien définis) qui pourraient être impliqués dans la méthylation des séquences virales après intégration. Ir : inversed repeat. 
2. Ensuitc, une dcuxièmc répression, elle aussi spécifiquc des ccllules EC, utilise une région situéc en aval du démarrage de la transcription, en dehors du LTR, du nucléotide +147 à +174 . Cettc région, appelée RBS (pour repressor binding site) [8] ou NRE (pour negative regulatory element) [9], recouvre en partie la région complémentairc de l'ARN de transfert prolinc qui scrt d'amorce à la transcriptase inversc au cours de la réplication du virus. La répression reste efficace quclles que soient la position et l'orientation de la séquence RBS. Elle pcut aussi agir sur d'autres promoteurs que celui du MoMLV. Cet élément a donc toutes les caractéristiques des silencers transcriptionnels $[8,9]$. Cependant, il restc à en découvrir la base moléculaire, qui, probablement, n'est pas très simple. Ainsi, la répression par le RBS, qui est encore obscrvée dans les conditions où l'ADN du gènc rapporteur n'est pas intégré au génome de la cellule (expression transitoire), dépend alors de la position de celuici dans le vecteur: le RBS n'agit pas s'il est déplacé de sa position naturelle (cn 3' du LTR, dans les séquences transcrites, figure I) en 5' du LTR (dans les séquences non transcrites) [9]. L'hypothèse avancée pour rendre compte de cette observation cst la suivante : le RBS exercerait son cffet répresseur par un mécanisme à deux niveaux. Lc premier, mis en évidence avec l'ADN non intégré, dépendrait strictement de la position (une répression au niveau de l'ARN n'est pas exclue) ; le second, mis en évidence avec l'ADN intégré, ne dépendrait pas de la position du RBS (une répression par méthylation de l'ADN est envisageable). Cette hypothèse d'un mécanisme à deux niveaux pourrait expliquer que plusieurs factcurs se fixent in vitro sur la région +147 à +174 .

3. Enfin, le promoteur minimal (la région du LTR qui contient les boîtes CAAT et TATA) a une activité réduite dans les cellules multipotentielles. L'activité dans les cellules EC est 10000 fois plus faible que dans les cellules différenciées [4]. Cela vaut particulièrement pour les plasmides intégrés au génome.

Ces observations ont été étendues aux rétrovirus recombinants [10]. Pour

$\mathrm{m} / \mathrm{s} n^{\circ} 2$ vol. 9, février 93 cela les rétrovirus ont été altérés dans les trois régions mentionnécs: la région - 354, - 304, qui fixe avec une moindre cfficacité que MoMLV lc factcur ECF-1 ; la région - 166, où un site de fixation de l'équivalent murin du facteur $\mathrm{Sp1}$ a été introduit; et la région $+147,+174$ (RBS), qui a été changée pour celle d'un virus mutant, dl 587 rev. L'expression de ces nouveaux rétrovirus n'est pas bloquée dans les cellules EC et ES : lc nombre de colonies obtenues en milieu sélectif avec les rétrovirus recombinants ainsi modifiés est du même ordre de grandcur sur les ccllules multipotentielles et sur les cellules différenciées. Ainsi, lorsque les trois niveaux de verrouillage du virus sont levés, celui-ci fonctionne efficacement. Ces rétrovirus représentent une alternative à une autre classe de rétrovirus, précédemment imaginée pour exprimer des gènes dans les cellules EC et ES, les rétrovirus à promoteur interne. Dans ccs rétrovirus, un promoteur non viral est placé en amont du gènc que l'on veut exprimer, rendant ce dernier indépendant du promoteur viral [11].

\section{Mécanismes de l'activation des provirus dans les cellules EC}

L'étude du blocage des rétrovirus murins dans les cellules EC a aussi été abordée après infection virale en utilisant des rétrovirus recombinants comportant un gène de sélection sous contrôle transcriptionnel des éléments du virus. Deux sortes d'informations ont pu être obtenues.

Premièrement, l'examen détaillé de la structure de plusieurs des provirus intégrés dans des clones de cellules multipotentielles se multipliant en milieu sélectif $[12,13]$ a confirmé la validité des observations précédentes. En particulier, beaucoup des provirus actifs dans les cellules EC ont soit une mutation au niveau du RBS, soit présentent une délétion complète de ces séquences. Cela confirme le rôle majeur de la région RBS dans le contrôle négatif de l'expression du rétrovirus recombinant. Les transcrits viraux, codant pour le gène de sélection, sont produits à partir du LTR. Cependant, au moins dans certains cas (peut-être même dans tous), outre ces modifications structurales du rétrovirus, il faut que celui-ci soit intégré à proximité d'un élément activatcur cellulaire actif [13]. Cela est apparu en comparant les efficacités d'expression dans les cellules EC des provirus reclonés en présence (forte cxpression) et en l'absence (faible expression) des séquences cellulaires adjacentes au point d'intégration.

Deuxièmement, l'examen dc la structure des autres provirus a montré l'existence d'un mécanisme pour lequel aucunc modification de la structure provirale, ni dans les régions mentionnées précédemment ni dans d'autrcs, n'est nécessaire. La comparaison des sites d'intégration de clones d'origine indépendante a abouti à un résultat remarquablc. Elle a permis de classer les clones en un petit nombre de familles, chacunc correspondant à une région particulière du génome où les rétrovirus intégrés sont actifs. Ainsi, dans la lignée $\mathrm{F} 9$, trois familles ont été décclées [12] ct, dans la lignée PCC4, unc famille majcurc différente des précédentes a pu être repéréc [14]. Cette observation pose un premier problème : comment les multiples blocages viraux décrits sont-ils contournés?

Plusieurs observations suggèrent quc l'cffet cssenticl de ces régions du génome sur le provirus ne réside pas dans le fait qu'elles soient dans un domaine chromatinien ouvert ou qu'elles aient un effet activateur ou conformationnel à distance sur le promoteur viral. En effet, ces hypothèses ne rendent nullement compte de ce que tous les rétrovirus intégrés dans une même région s'y trouvent toujours orientés dans le même sens $[12,14]$. Une autre explication devait donc être recherchéc.

Le mécanisme d'activation des provirus tel qu'il vient d'être établi dans un article récemment publié [14] est résumé dans la figure 2, p. 194. Lcs régions du génome qui permettent l'expression des provirus abritent un gène qui est exprimé dans les cellules EC (figure $2 A$ ). Les provirus sont intégrés dans le premicr ou l'un des premiers introns du gène actif (figure $2 B$ ). Le provirus est transcrit à partir du promoteur de ce gène. La transcription ignore le signal de 


\section{RÉFÉRENCES}

11. Rubenstein J, Nicolas JF, Jacob F. Construction of a retrovirus capable of transducing and expressing genes in multipotential embryonic cells. Proc Natl Acad Sci USA 1984; 81: 7137-40.

12. Barklis E, Mulligan RC, Jaenisch R. Chromosomal position or virus mutation permits retrovirus expression in embryonal carcinoma cells. Cell 1986; 47 : 391-9.

13. Taketo M, Shaffer DJ. Deletions in a recombinant retrovirus genome associated with its expression in embryonal carcinoma cells. J Virol 1989; 63 : 4431-3.

14. Bonnerot C, Legouy E, Choulika A, Nicolas JF. Capture of a cellular transcriptional unit by retrovirus : mode of provirus activation in embryonal carcinoma cells. $J$ Virol $1992 ; 66$ : 4982-91.

15. Peabody DS, Berg P. Terminationreinitiation occurs in the translation of mammalian cell mRNAs. Mol Cell Biol $1986 ; 6$ : 2695-703.

16. Stamatoyannopoulos G. Human hemoglobin switching. Science $1991 ; 383$ 252-383.

17. Linial M. Creation of a processed pseudogene by retroviral infection. Cell $1987 ; 49$ : 93-102

18. Hu WS, Temin HM. Rctroviral recombination and reverse transcription. Science $1990 ; 250$ : 1227-33.

19. Swanstrom R, Parker RC, Varmus HE, Bishop JM. Transduction of a cellular oncogene: the genesis of Rous sarcoma virus. Proc Natl Acad Sci USA 1983; 80 : 2519-23.

20. Stuhlmann H, Dieckmann M, Berg P. Transduction of cellular neo mRNA by retrovirus-mediated recombination. $J$ Virol $1990 ; 64: 5783-96$.

21. Varmus $\mathrm{H}$, Brown P. Retroviruses. Mobile DNA Washington: American Society for Microbiology, 1989 : 53-92.

22. Weiss R, Teich N, Varmus H, Coffin J. RNA tumor viruses. Molecular Biology of Tumor Viruses, $2^{\text {nd }}$ ed. 2. Supplements and Appendixes. New York : Cold Spring Harbor Laboratory, 1985: 1-1222.

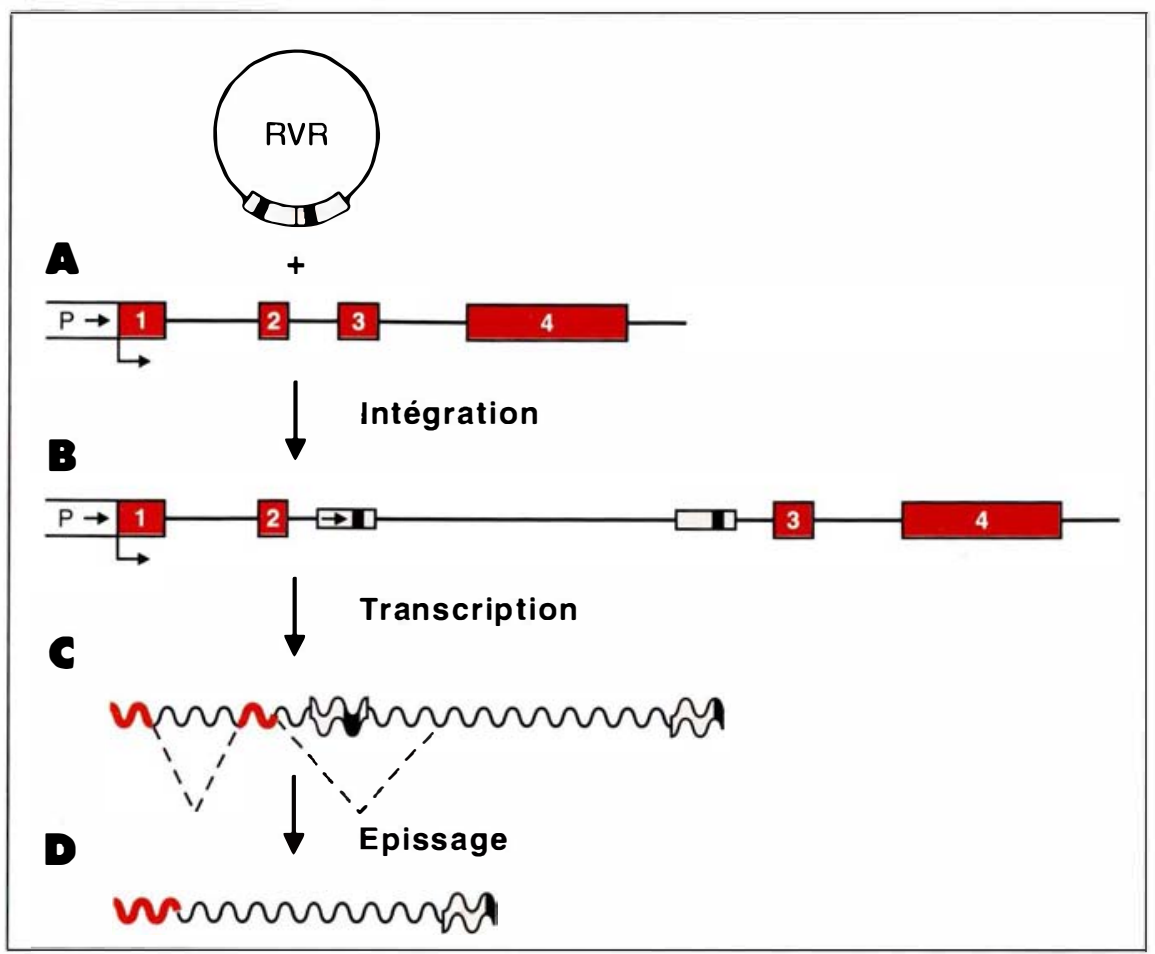

Figure 2. Mécanisme d'activation des provirus dans les cellules multipotentielles. A et B. Intégration du virus dans l'intron d'un gène actif. Le cercle représente le rétrovirus recombinant (RVR) avant intégration au génome. Les LTR sont représentés par les rectangles noirs (R) et roses (U3 et U5). Le gène cellulaire est schématisé en $A$ par des boîtes : la boîte $P$ (blanche) contenant le promoteur et les boîtes 1 à 4 (rouges), correspondant aux exons, séparés par les introns (lignes simples horizontales). B. Structure du provirus dans le gène cellulaire. Le promoteur cellulaire $(P)$ est actif mais non le LTR viral. $C$ et $D$. Structure de I'ARN produit. $C$. ARN, avant épissage, produit à partir du promoteur cellulaire $(P)$ capturé par le rétrovirus. $D$. ARN hybride cellulaire-viral, après épissage.

polyadénylation de la région $\mathrm{R} d \mathrm{du}$ LTR 5'. Un ARN hybride (cellulaire et viral) est synthétisé (figure 2C). Il est ensuite épissé à partir du site donneur de l'exon cellulaire jusqu'à un site accepteur viral cryptique (le site accepteur d'épissage qui sert à fabriquer l'ARNm du gène de l'enveloppe est absent de la construction). De ce fait, seule une partie du génome du provirus se retrouve dans l'ARN mûr (figure $2 \mathrm{D}$ ). Dans le cas de la région détectée dans les cellules PCC4, ce site accepteur viral est localisé dans les séquences du virus de Moloney. Il est précédé d'un consensus " point de branchement*" qui n'avait pas été reconnu jusqu'à présent. L'ARN hybride est polyadénylé à la dernière base de la région $\mathrm{R}$ du LTR 3'.

Mais alors, pourquoi y a-t-il si peu de régions piégées par le rétrovirus recombinant? Plusieurs raisons peuvent être évoquées. Il faut que le promoteur cellulaire soit insensible aux mécanismes qui répriment le promotcur viral. De ce point de vuc, il est intriguant de constater que tous les gènes piégés ont des promoteurs très riches en dinucléotides $\mathrm{GC}$, comme la plupart des gènes à expression ubiquitaire dits de fonction de ménage. Il faut aussi que le signal de polya-

\footnotetext{
* Séquence au niveau de laquelle s'établit, lors de l'épissage, la liaison entre le 5' phosphate de l'acide guanylique en 5' de l'intron et l'hydroxyle 2' d'un résidu adénylique localisé quelques dizaines de bases en amont de l'extrémité 3' de l'intron, c'est-à-dire du site accepteur d'épissage. C'est cette liaison qui confere à l'intron sa structure en lasso, caractéristique des réactions d'excision-épissage.
} 
dénylation du LTR 5' soit ignoré. Enfin, la traduction de l'ARN codant pour le gène de sélection doit pouvoir sc faire efficacement. Cette dernière condition implique que le virus soit intégré dans un des tout premiers introns d'un gène, en aval d'un cxon, de préférence non codant. Ainsi, l'épissage de l'ARN cellulaire ct du LTR place l'AUG du gène rapportcur en première position, condition qui favorise la traduction [15]. Il cst étonnant de constater qu'il n'y a pas plus souvent activation du promoteur minimal du virus par des éléments activateurs du génome. Cela illustre peut-être le fait que les cellules EC utiliseraient peu d'éléments activateurs et, plus généralement, auraient pcu de gènes actifs. En conséquence, il y aurait peu d'activateurs disponibles dans cet état cellulairc particulier. De plus, il est possible que l'élément silencer du virus soit dominant par rapport aux activatcurs. Il y a d'ailleurs des précédents à cettc dernière situation : c'est par un mécanisme de répression domi- nant les effets activateurs du LCR que se font les commutations d'un gènc à un autrc dans le locus $\beta$ globine au cours de l'embryogenèse [16].

\section{Un nouveau mécanisme de capture de séquences cellulaires par les rétrovirus?}

De plus ce mécanisme d'activation des rétrovirus permet d'entrevoir de nouvelles stratégies utilisées par les rétrovirus pour détourner, à leur profit, l'information du génome.

Ainsi, même dans les conditions où lc rétrovirus est silencieux, certains de scs gènes sont éventuellement transcrits par détournement d'un promoteur cellulairc. Cela pourrait rendre comptc de maladies associées aux rétrovirus plus complexes que MoMLV, tels BLV (bovine leukemia virus) ou HTLV -1 et -2. En particulier, certains des gènes impliqués dans le contrôle du virus (tax et rex), ct dont l'expression perturbe le con- trôle de certains gènes de l'hôte, pourraient ainsi être transcrits indépendamment du virus. I a possibilité de ce mécanisme de transcription et de traduction de ces gènes viraux par la cellule hôte est renforcée du fait de la présence de sites accepteurs d'épissage naturcls (ccux utilisés pour produire les ARNm tax et rex) dans ces virus.

En outrc, la mise sous contrôle ccllulairc du provirus pourrait être à la base d'un processus de capture par le virus de matériel cellulaire situé en 5' du sitc d'intégration (figure 3). La capture s'effectuerait à la suitc du cocmpaquetage d'unc molécule d'ARN hybride et d'unc moléculc d'ARN viral dans une particule rétroviralc. Ces virus hérérodimères serviraient cnsuite de matrice pour fabriquer, au cours de la transcription inverse, un provirus contenant à la fois des séquences cellulaires et virales. On sait déjà que l'empaquetage dans les particules rétrovirales d'ARN dépourvu des signaux viraux d'cmpaquetage peut se produirc [17] et que

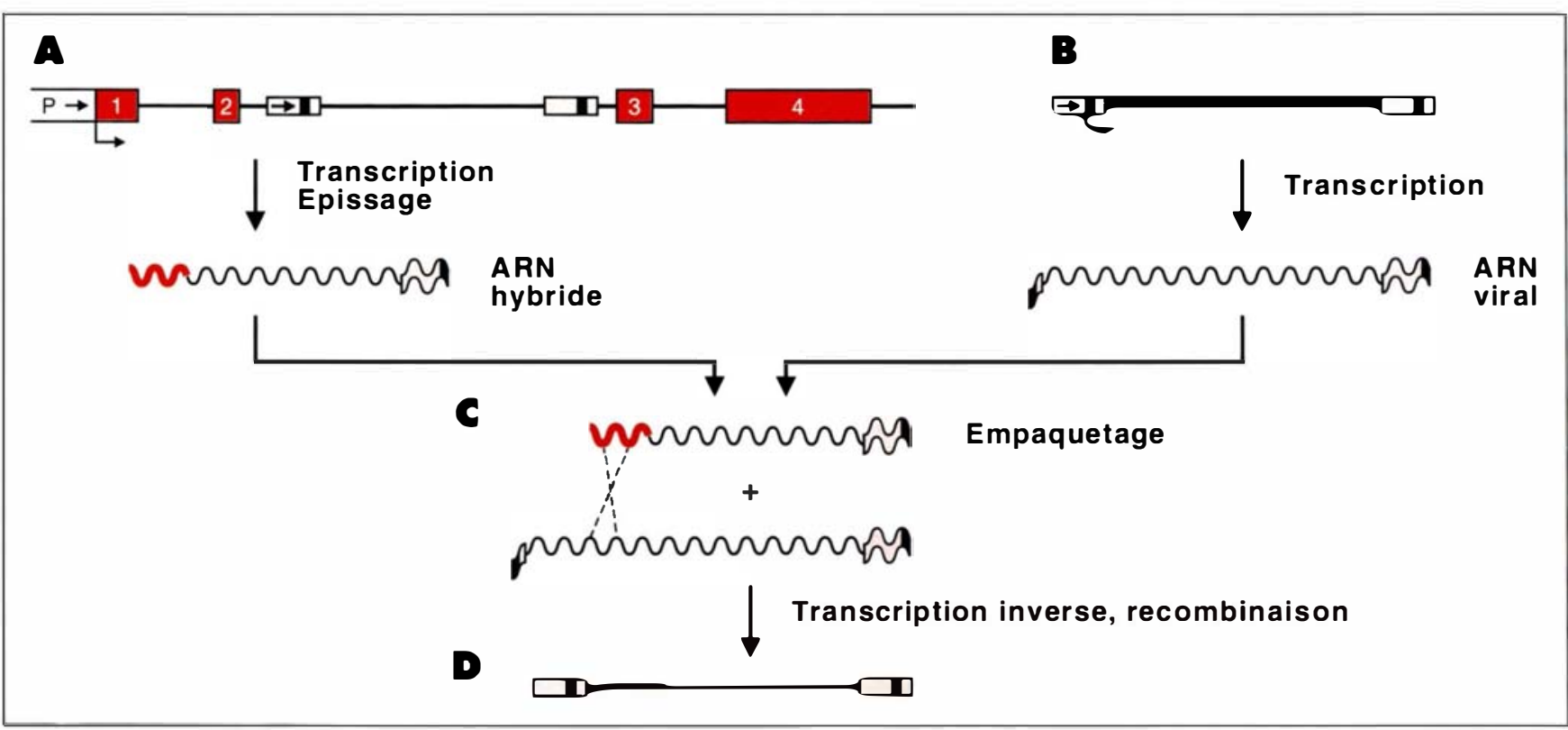

Figure 3. Mécanisme d'annexion, par les rétrovirus, d'exons cellulaires localisés en $5^{\prime}$ du site d'intégration. A. Structure de I'ARN hybride cellulaire-viral produit à partir du promoteur cellulaire $P$. B. ARN viral produit par un retrovirus sauvage (dans la même cellule). C. Coempaquetage de l'ARN hybride et de I'ARN viral dans le rétrovirus (" hétérozygote"). D. Au cours de la transcription inverse, une recombinaison et/ou des changements de matrice de la polymérase conduisent à la production d'un provirus recombiné. 


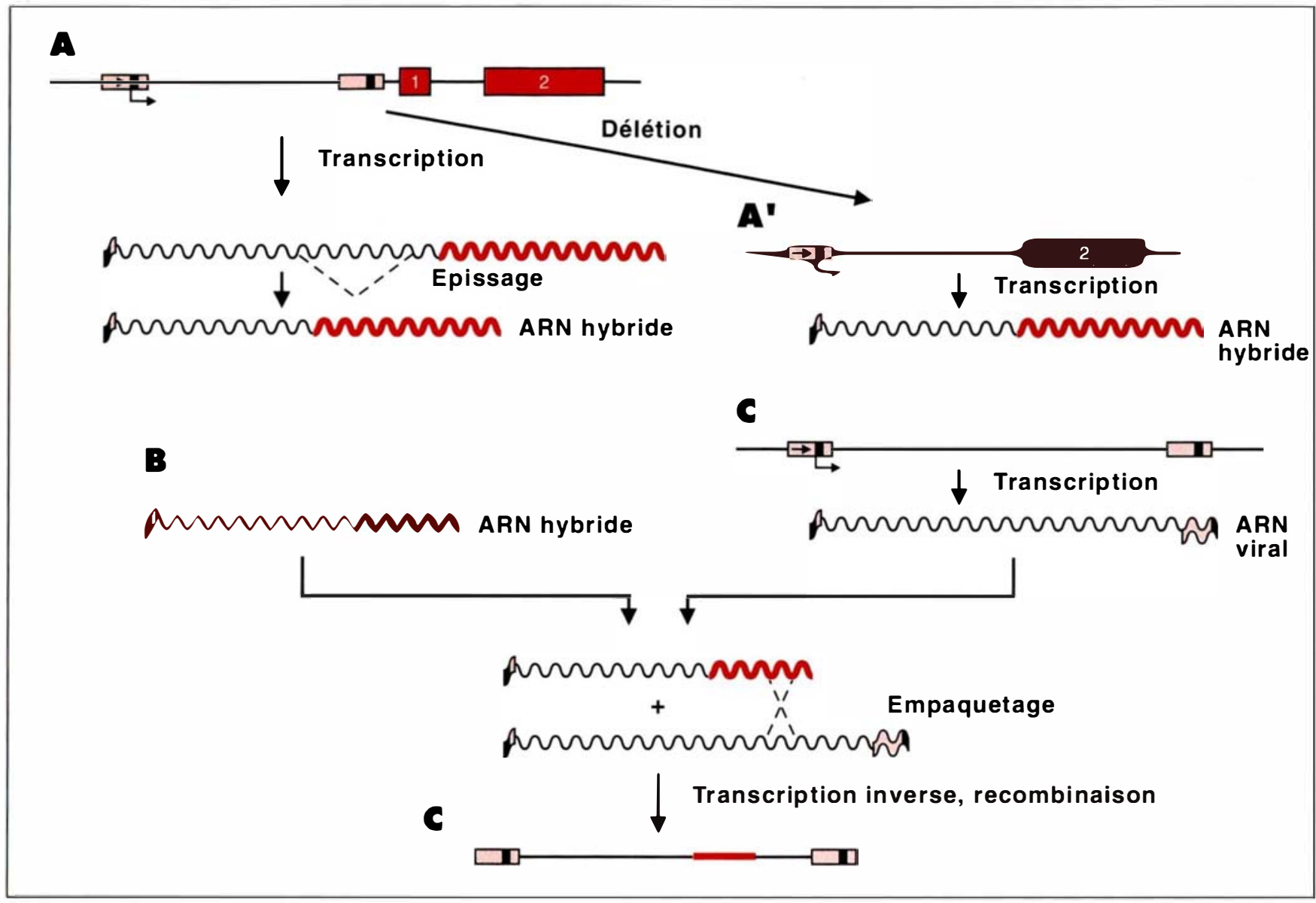

Figure 4. Mécanisme d'annexion, par les rétrovirus, d'exons cellulaires localisés en 3' du site d'intégration proposé par Swanstrom et al. [19]. A. Le provirus est intégré en amont des exons 1 et 2 lcodant éventuellement pour un oncogène cellulaire). Un ARN hybride regroupant sur la même molécule les séquences virales et cellulaires peut être produit lorsque le signal de polyadénylation du LTR 3' est ignoré et que l'ARN est épissé comme indiqué. $A^{\prime}$. Alternativement, une délétion du LTR $3^{\prime}$ joint les séquences virales et cellulaires dans une même unité de transcription qui produit un ARN hybride. B. ARN hybride. C. ARN viral produit par un rétrovirus sauvage ldans la même cellule). D. Coempaquetage de l'ARN hybride et de l'ARN viral dans la particule virale (" hétérozygote "). E. Au cours de la transcription inverse, une recombinaison et/ou des changements de matrice de la polymérase conduisent à la production d'un provirus recombiné ayant capturé les exons 1 et 2 (éventuellement oncogéniques).

la transcriptase inverse change fréquemment de matrice au cours de la réplication virale [18]. Cela augmente la vraisemblance du mécanisme de capture. Une variante de ce schéma a été produite dans le passé pour expliquer la capture des oncogènes par les rétrovirus (notamment chez les aviaires), à ceci près qu'alors, c'est le promoteur virale du LTR 5' qui assure la capture de l'oncogène situé en 3' du site d'intégration (figure 4) [19]. Bien que ce nouveau type de capture de séquence 5' reste à démontrer plus précisément, sa vraisemblance est suggérée par certaines

ont établi que la présence d'un seul LTR est suffisante pour que les événements de recombinaison entre les ARN coempaquetés dans la particule virale aient lieu [20]. Cela constituerait une nouvelle indication de l'action des transposons (dont les rétrovirus ne sont qu'un des exemples) dans le maintien de la fluidité des génomes [21].

Mais, finalement, ce mécanisme pourrait aussi avoir été exploité, en retour, par les cellules de la lignée germinale. En effet, un des gènes qui peuvent être transcrits et traduits dans les cellules multipotentielles par le mécanisme décrit ci-dessus est celui de l'enveloppe du rétrovirus qui est normalement produit à partir d'un ARNm viral épissé. Or, lorsque le produit du gène de l'enveloppe est exprimé à la surface d'une cellule, l'infection par un nouveau rétrovirus n'est plus possible, les récepteurs viraux se trouvant bloqués [22]. Cette circonstance permettrait de limiter la propagation des rétrovirus dans l'espèce, et ainsi de diminuer la charge génétique due aux mutations par insertion. Ne serait-ce pas, d'ailleurs, cette nécessité qui rendrait aussi compte de la multitude des blocages que les cellules de la lignée germinale imposent aux rétrovirus? 


\section{Summary}

Repression and activation of murine retroviruses in multipotential cells

The expression of murine leukemia virus (MuLV) in embryonal carcinoma (EC) cells is blocked. The blockage is due to a multiplicity of negative controls of viral transcription : a negative element within the viral enhancers and others mapped 5' to them as well as a strong silencer in the leader sequence of the virus. Despite these negative controls, rare cases in which viral expression occurs have been detected when selective methods have been used. The complete analysis of the mechanism of activation establishes that viral transcripts are initiated from a upstream host-cell promoter and are spliced from a host donor to a cryptic viral acceptor in the MLV sequences. These results point to a mechanism by which retroviruses can acquire host sequence by 5' annexation of exons. It also indicates that retroviruses can be transcribed from upstream nonviral elements.

TIRÉS A PART

J.-F. Nicolas. 